\title{
EXPLORATION OF PRACTITIONER EXPERIENCES OF FLEXIBILITY AND TRANSPARENCY TO IMPROVE BIM-BASED MODEL CHECKING SYSTEMS
}

\author{
SUBMITTED: April 2019 \\ REVISED: November 2021 \\ PUBLISHED: December 2021 \\ EDITOR: Robert Amor \\ DOI: $10.36680 /$ j.itcon.2021.055 \\ Peter N. Gade, Lektor, \\ University College Northern Denmark, Department of Architectural Technology and Construction \\ Management / Aalborg University, Department of Civil Engineering \\ pega@ucn.dk
}

Kjeld Svidt, Associate Professor,

Aalborg University, Department of Civil Engineering

ks@civil.aau.dk

SUMMARY: The use of BIM-based Model Checking (BMC) has the potential to improve building design processes by enabling the automation of building assessments. However, only a few BMC systems are being used in the building design practices. The limited use has been identified to be related to socio-technical challenges that have so far not received much attention in research regarding BMC systems. To explore these challenges, a Design Science Research methodology was used to design a BMC prototype to improve the socio-technical challenges of BMC systems, specifically challenges of transparency and flexibility. The prototype was tested with practitioners to investigate aspects of BMC systems that potentially hinder its use. The results were used to discuss the potential for more practical application of BMC systems in design practices to obtain the benefits of providing a faster, more consistent, and more precise assessment of buildings.

KEYWORDS: BIM-based Model Checking, BIM, Flexibility, Transparency.

REFERENCE: Peter N. Gade, Kjeld Svidt (2021). Exploration of practitioner experiences of flexibility and transparency to improve BIM-based model checking systems. Journal of Information Technology in Construction (ITcon), Vol. 26, pg. 1041-1060, DOI: 10.36680/j.itcon.2021.055

COPYRIGHT: () 2021 The author(s). This is an open access article distributed under the terms of the Creative Commons Attribution 4.0 International (https://creativecommons.org/licenses/by/4.0/), which permits unrestricted use, distribution, and reproduction in any medium, provided the original work is properly cited. 


\section{INTRODUCTION}

A fundamental aspect of designing buildings are the designer's ability to assess buildings performance. However, we know that the manual process is prone to be inefficient and a cause to declining productivity but by automating the assessment BIM-based Model Checking (BMC) can be applied to improve this assessment by potentially making it faster, more precise, and more consistent (Amor and Dimyadi 2020). BMC is related to the many terms describing the process of automatically assessing BIM model performance and is also known as rule checking, model validation, quality checking, automated code checking, BIM checking and code compliance checking and many more. The term BMC was proposed by Hjelseth (2015) as a "joint term" to indicate the commonalities between these many other. BMC was catagorized into four sub-types: validation checking (a comparison of the information in the BIM model against pre-defined rules), content checking (the right content and quality of information in the BIM model), smart objects checking (BIM objects that act upon predefined logic) and design options checking (which suggests alternative solutions using a knowledge database).

BMC has been a focus of research and development for years, but its practical use throughout the years been reported as limited (Dimyadi and Amor 2013, Refvik et al. 2014, Beach et al. 2020). While, there exist many BMC solutions a recent study by Beach et al. (2020) indicated that "there has been no meaningful adoption, despite the increasing maturity". The adoption of BMC is considered by some researchers slow and problematic (Refvik et al. 2014, Beach et al. 2020). Beach et al. 2020 found that some of the reasons to be related to lack of shared open standards for regulation clauses, not tools to offer a complete ability of precheck for compliance prior to formal submissions, cultural resistance. Ahmed (2018) found that the main barrier for using BIM in general are social and habitual resistance for change. This has also been substantiated by different authors in regards of BMC. Beach $e t$ $a l$. (2020) argues that is related to a wide range of different obstacles that not are of technical, but commercial, political and social issues. Similarly, argues, Refvik et al. (2014), that a main challenge is of a socio-technical nature. "The technology is mature and available; it is the soft human aspects of organization, culture, and adoption of the technology that are the real challenges." (Refvik et al. 2014, p. 58).

So far, there has been limited interest in investigating the socio-technical issues of BMC, but there are indications that extrinsic socio-technical challenges in the relationship with the technology and the designer's practices. So far, there do not exist much research exploring the socio-technical issues in depth. However, it is acknowledged that there is a need for the user's ability to understand and adapt the systems to their context often talked about as the need of flexibility/transparency with issues of hard-coding or black-boxing (Dimyadi and Amor 2013, Refvik et al. 2014, Preidel and Borrmann 2016, Kim et al. 2018, Fan et al. 2019). Fan et al. (Fan et al. 2019) argues that there is a lack of flexible rule manipulations and need for better tractability. Both Fan et al. (Fan et al. 2019) and Kim et al. (2018) have attempted to create solutions to solve these issues but did not emphasize any empirical ingestions for the problems experienced in practice. Preidel and Borrmann (2015a) explain that the lack of transparency detaches the user from the assessment process and creates uncertainty related to the correctness of the assessment, which can lead to, e.g., legal issues. The practitioners need to adapt the information processes in order to ensure that the assessment is conducted according to their unique context (Reinhardt and Matthews 2017).

While BMC is a popular research topic, there is a lack of interest and research concerning the socio-technical issues while researchers indicate socio-technical problems. The focus is to solve rather than understand the problems. The lack of dissemination is by some researchers related to the uneasy relationship between many researchers in the engineering domain with, e.g., social science perspectives. yet such perspectives can open for new understandings and solutions for the field (Miettinen and Paavola 2014, Koch et al. 2019). However, currently, there are either very limited to no formalized scientific dissemination of practitioners experiences of using BMC systems (Preidel et al. 2017). Instead, the identified phenomena of the user's rejection of the systems are typically reduced to cultural resistance to habitual change and can be constituted as a technological deterministic view of technology, that the technology is developed independent from social concerns. An alternative to such view has been proposed by Orlikowski (1992) that states that by better understanding the technology and the environment in which it is used, it is possible to identify practices that both constrain and facilitate the development and deployments of technology. Such perspective can potentially contribute to the domain of BMC in order to expand on these illustrious socio-technical issues and lead to better systems that are more applicable for practitioners.

In this article, we strive to get a better understanding of the relationship between BMC systems and the designer's practice. A better understanding of this relationship can unfold characteristics important for the relationship between BMC-systems and practitioners in successful adaption and better development of BMC systems. The 
results of such an investigation can potentially identify new topics for future research to help inform new more "adoptable" BMC systems. This leads to the research question: What characteristics are important for the functionality and development of BMC systems experienced by the practitioners?

\section{METHODOLOGY}

The research questions are aimed towards exploring socio-technical challenges of BMC systems. This exploration follows a dualistic perspective of technology that both entail that technology is produced for and by the practices that adopts it. Orlikowski (1992) described the duality as a flux between the objective reality (the actual functions of the systems) and the socially constructed product (the uses that the users can imagine and apply in their practices). This duality necessitates a view of technology as interpretively flexible because individual users are subject to their context. In other words, the use of a system is based on the individual user's context (e.g., education, project, company). Without recognizing that BMC systems need to accommodate existing practices, there is an inherent risk that unintended challenges will affect the practices that the systems aim to improve. "The relationship between technology and society cannot be reduced to a simplistic cause-and-effect formula. It is, rather, an 'intertwining', whereby technology does not determine but operates, and is operated upon in a complex social field" (Murphie and Potts 2017). This relationship is explored using design science research methodology.

\subsection{Design Science Research}

For such investigation, a Design Science Research (DSR) methodology was applied. DSR is a pragmatic methodology that emphasizes the development and evaluation of an artifact (Hevner et al. 2004), in this case, a BMC prototype. According to Hevner et al. (2004), DSR is suitable in information systems domains that are subject to changing and unstable requirements and constraints that require complex interactions among the subcomponents of the problem (the design practice) and its solution (BMC systems) and are dependent on the users' cognitive abilities (like creativity) and their social interactions. These characteristics are found in the domain of BMC and the construction industry, which is characterized as being unstable, dynamic and unexpected (Bertelsen, 2003; Wood, Piroozfar, and Farr, 2013) and requires complex social interaction (Cicmil and Marshall 2005, Kazi and Koivuniemi 2006).

DSR focuses on ensuring relevance and rigour. The relevance is ensured by attending to business needs by ensuring that the artifact (BMC system) is applicable in the appropriate environment (design practice). Rigour is ensured by building the artifact on foundations (e.g., theories, methods, and instruments) and methodologies (e.g., data analysis techniques, measures) that are applicable for the domain of research. The business needs and applicable knowledge are used to both develop and evaluate the performance of the artifact. The results of this process are used to disseminate insights that can be used to improve the functional performance of BMC systems. It is therefore not a study that sets out to focus on the specific individual aspects of BMC like interpretation, implementation, and validation, or the content of the BIM model or the prototype itself. Instead, it provides a holistic account of a BMC prototype test that is used to highlight and discuss the socio-technical characteristics that potentially impair the functional performance of BMC systems in certain enviornments.

DSR has previously been used in the domain of BIM (Sacks et al. 2017, Schimanski et al. 2019). The previous uses of DSR was aimed towards developing artefacts for business processes with an emphasis of evaluating the prototype with industry partners. However, the main application of DSR has been artefact centred in developing e.g., prototypes and to a lesser degree the evaluation of the prototype. For example, in Sacks et al. (2017) s study, three professionals are invited to evaluate the prototype, however, this process remains very opaque and limited. Similarly, Schimanski et al. (2019) is also emphasising less on the evaluative aspects of DSR.

In this study, the focus is to investigate the extrinsic aspects of BMC use that cause challenges to proper adoption of the technology. It also follows, the DSR pragmatic foundation of research where it is the changes in practices that are of interest rather than how e.g., the artefact is built. The development and practical application of the artefact is used to externalize "soft" and challenging notions of BMC adoption. Moreover, it also accepts the notion that a universal optimal or correct BMC prototype is not achievable but is dependant of many factors based in the individual environments. This prototype constitutes an effort to accommodate issues previously highlighted in the literature regarding transparency and flexibility which will be theoretically accommodated in the prototype and give insights to how such prototype works in a certain environment. 
According to the DSR methodology to develop and test an artifact, the following steps were conducted:

1. Identification of requirements for the prototype

Theories were proposed to improve the existing identified issues to allow better adaptation of BMC systems into practice.

2. Creation of a prototype based on the requirements

A prototype was created using readily available software tools that can accommodate the functional requirements identified in the previous step.

\section{Prototype test}

The prototype was tested by practitioners and compared with their traditional approach to assessing.

\subsection{Identification of requirements for the prototype}

The requirements for the BMC prototype are based on the basic BMC functionalities defined by Eastman et al. (2009) which include BIM model preparation, rule execution and report checking results. Also, we include functionalities to accommodate the socio-technical issues highlighted in the introduction, which include transparency and flexibility. Functional requirements to improve these issues are presented in the next sections.

\subsubsection{Black-boxed BMC-systems}

BMC systems often consist of comprehensive and complex automated processes that are challenging for the users to comprehend (Hjelseth 2015, Preidel and Borrmann 2016, Ghannad et al. 2019, Amor and Dimyadi 2020). So as not to overwhelm the users, they then tend to black-box most of the processes, leaving a set of specific parameters open for adjustment. However, as Amor \& Dimyadi (2021) argues, it is problematic for user validation of BMC systems. Moray et al. (2000) argue that the more comprehensive and complex the information processes are, such as BIM-based model checks, the less able users are to comprehend the processes. Cornelius and Borrmann (2016) argue that if users are not able to ensure the correctness of the BMC systems, this will lead to a lack of trust. A lack of trust often results in the systems being rejected, and users will resume previous methods of working (Hoffman et al. 2013). In relation to BMC systems that can be used to assess the room heights of a building, where the user is unable to scrutinize the process of how the assessment is conducted, the user is not able to ensure that the quality is sufficient. The quality is defined by the quality standards of the contexts where the system is used, based on, e.g., localized standards, laws, technology and culture (Reichert and Weber 2012).

Transparency has to be improved to allow the users of BMC systems to scrutinize the information processes (e.g., their quality) to increase trust (Hoffman et al. 2013). Transparency can increase the user's understanding of how information is processed and increase the user's ability to predict how it will be processed in the future. For example, a programmer that developed a piece of software herself have full transparency of the information processes and can predict the outcome of the software's use with high certainty. One of the possible solutions to improve transparency is the use of Visual Programming Language (VPL). Using VPLs for BMC systems can improve the communication of the information processes between the system and the users, thereby increasing the transparency(Preidel and Borrmann 2015b, Häußler et al. 2021). The improvements enabled by visual programming are that it makes use of higher-level operators such as nodes to conduct operations that are presented graphically. While the visual programming can increase transparency, it also requires sound management of how it is applied. The poorly designed code in VPL makes it difficult for users to understand the information process and therefore requires structure, like colouring, commenting, grouping and modularisation (Green and Petre 1996).

Preidel and Borrmann (2017) proposed the modularisation of the visual programming semantics and ontology. In this article a focus will be put on a higher-level structure and modularisation due to the use of existing visual programming software that already provides a low-level structure (semantics and ontologies related to, e.g., the operations that nodes conduct). The structure used in this article will follow a hierarchal structure based on the fundamental steps of automation suggested by Parasuraman, Sheridan \& Wickens (2000):

- Acquisition - Registration of input data, e.g., registration of the right definition of room height from a BIM model.

- Analysis - Analysis of input data through algorithms, e.g., calculating the average room height.

- Decision - Selecting a decision based upon the analysis, e.g., assigning the right score to the average room height.

- Action - Conducting the correct action based on the decisions, e.g., submitting the score to a score database. 
Structuring the checks conducted by a BMC system into horizontal axis illustrating the checking progress (sequence of operations) and a vertical axis illustrating the levels of abstraction (higher level less detail, lower level more detail). This structure will assist the users in identifying specific parts of the information processes. If the user knows what aspect of the check he needs to scrutinize, e.g., what information the room height is based on, the user can identify that such information will be placed in the Acquisition node. The operations needed to conduct the Acquisition would include gathering the right data from the BIM model and assembling it for the Analysis, e.g., the process of obtaining the right room height data. The structuring of the operations in such a hierarchy is presented in FIG. 1.

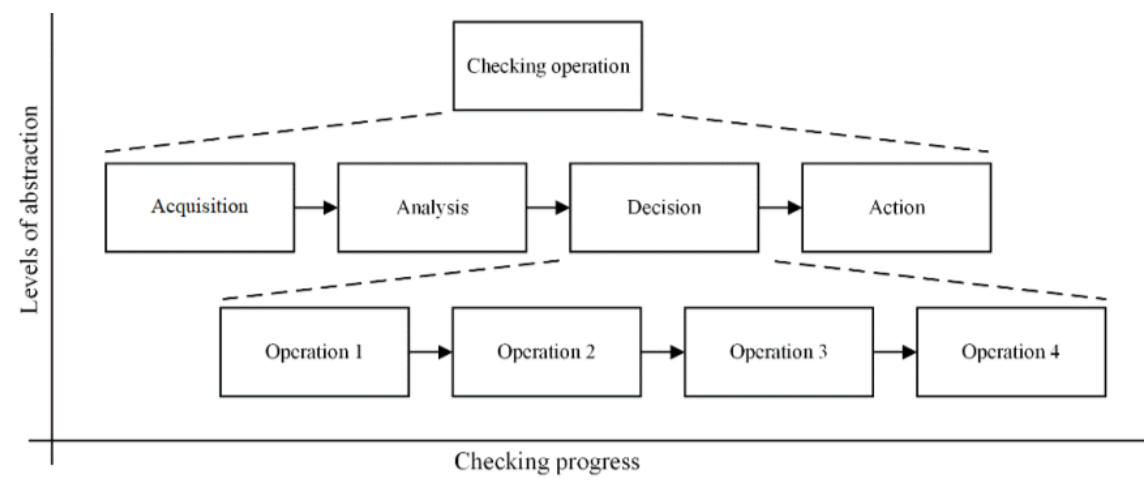

FIG. 1: Hierarchy of nodes

\subsubsection{Hard-coded BMC systems}

The majority of BMC systems (and information systems in general) are hard coded (Preidel and Borrmann 2017, Fan et al. 2019, Ghannad et al. 2019, Nawari 2019). Bell (1973) argues that the reason to why developers tend to use this approach is because it is efficient for the developers to rapidly execute their instructions of the system. However, this also embed a disadvantage for the users because they must accept or circumvent the developer's processes. Findings (Preidel and Borrmann 2017, Fan et al. 2019, Ghannad et al. 2019, Nawari 2019) indicate that there are issues related to the amount of hard-coding in BMC systems, which can indicate that the environment of BMC use requires more change than the current systems allow. Reichert and Weber (2012) argue that information systems are subject to different types of change. For a system to provide relevant feedback (e.g., to assess a building design using the latest building codes), it needs to adapt to such changes. The changes arise from both external and internal drivers of change. The external drivers are the changing business and legal context, new technology and system optimization due to organizational learning. The internal drivers are due to issues related to the development of a system, such as technical problems. The issue with BMC arises due to the different environments in which it is used. Each environment calls for a different adaptation to the changes, and the users must ensure that the BMC system processes the information with sufficient quality.

While some operations related to BMC are well-structured and highly repetitive, others are knowledge-intensive and highly dynamic. The latter are difficult to translate to fit all needs and contexts, and imbue the translated processes with great complexity and comprehensiveness, which leads to difficulties of both maintaining and using it in practice (Reichert and Weber 2012). Research regarding the changes that users of BMC systems make is limited, and not much is known about what changes are required regarding effective practical use. However, to better adapt to the different practices, Reichert and Weber (2012) argue that it is necessary to implement a series of functionalities in systems that are subject to major change. To improve the flexibility of systems, they suggest considering the following:

\section{Separation between the logic and the data objects}

Such separation will provide an additional architectural layer of the information processes that enhances the maintainability and traceability.

2. Separation between run- and build-time

Providing a system architecture with a separation between run-time and build-time components emphasizes the user's possibility to change specific operations according to the specific context. The build-time components allow specialists to ensure certain aspects of the information processes' validity and permit continuous optimization. 


\section{Loosely specified processes}

When an information process is very comprehensive and detailed, it increases the chance that it is difficult to adapt. When it is loosely specified, an emphasis is put on making it as simple as possible. While it may not accommodate all situations of use, it provides the users with the ability to defer decisions made in the processes.

\section{Exceptions}

Providing the user with the ability to deal with exception handling provided in the system code that will allow the user to adapt to the dynamic environments where a system is used.

\section{Performance analysis}

Providing analysis of the information processes along with other performance insights that can encourage organizational learning to support the improvement of existing processes for both run-time and buildtime users.

\subsection{Creation of a prototype based on the requirements}

The BMC prototype is created to accommodate the proposed requirements specified in the previous sections, including transparency, flexibility and BMC system functionalities in relation to validation checking.

\subsubsection{Prototype architecture}

The prototype is based on three software components to accommodate the requirements: Revit, Dynamo, and Tableau. These software components were selected to accommodate the functional requirements (in TABLE 1) and based on familiarity with the industry where the testing was conducted. Autodesk Revit is the most used BIM authoring system in Denmark and was selected for BIM authoring. Revit has a visual programming software component integrated named Dynamo (Dynamo BIM 2018) also used in the prototype. Dynamo can use information from the BIM models created in Revit and allows manipulation of the information through nodes and wires. Nodes are objects that perform operations, and wires connect those nodes. Dynamo contains a set of predefined nodes that can conduct basic operations, and it also allows the user to create customized nodes. Dynamo can be accessed either through the Dynamo player or as separate software. The Dynamo player is useful to execute the operations specified in Dynamo but does not present an overview. The data visualization software Tableau Desktop (Tableau 2018) was used to provide performance feedback to the user based on the BIM-based model checks conducted in Dynamo. The BMC-prototype architecture is illustrated in FIG. 2.

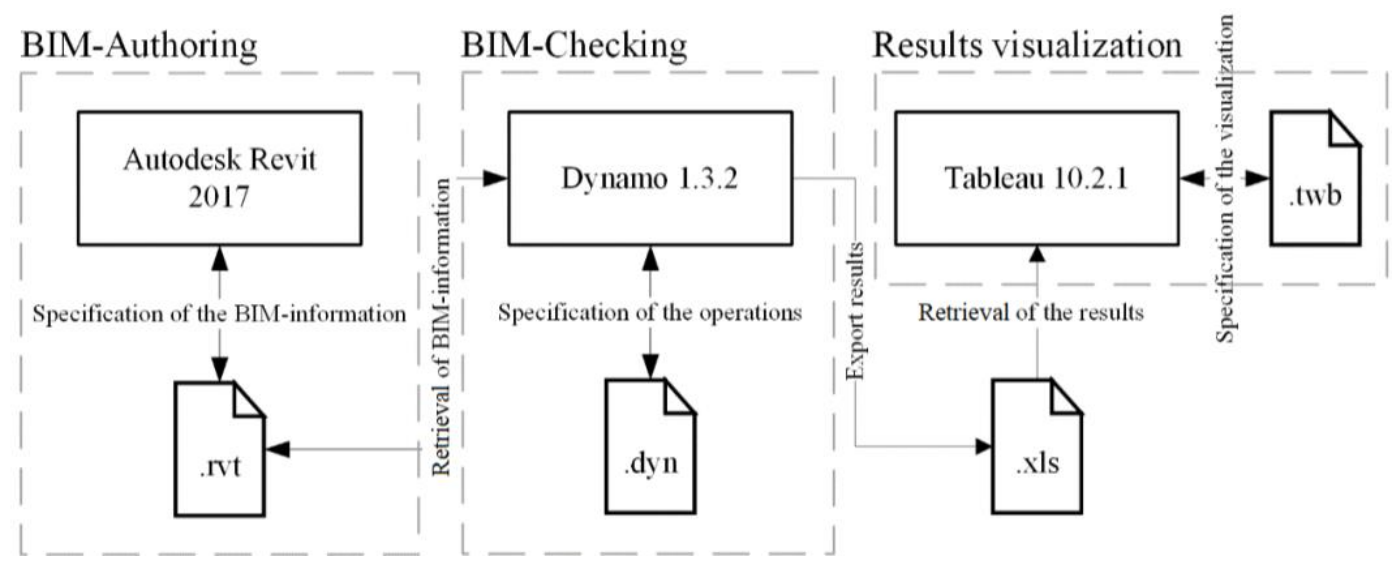

FIG. 2: The BMC-prototype architecture

The visual programming interface Dynamo allows both the run-time user and build-time user to achieve a visual representation of the operations of BMC. The prototype architecture is file-based and uses proprietary file formats for the sub-systems. The information from the project used in Revit is stored in .rvt. Dynamo uses .dyn to store the dynamo scripts and can export the results to .xls used by Tableau. Tableau stores its visualizations in .twb. Each of the software components fulfills a role related to the functional requirements (detailed in chapter 2.1) specified in TABLE 1. 
TABLE 1: Functional requirements of the prototype

\begin{tabular}{|c|c|c|c|}
\hline Theme & Requirement & Function & $\begin{array}{l}\text { Software } \\
\text { component }\end{array}$ \\
\hline \multirow{4}{*}{$\begin{array}{l}\text { BIM-based } \\
\text { Model } \\
\text { Checking - } \\
\text { Validation } \\
\text { Checking }\end{array}$} & Rule interpretation & $\begin{array}{l}\text { Formulation of rules in a } \\
\text { programming language }\end{array}$ & Dynamo \\
\hline & BIM-model preparation & Extraction of BIM-model information & Revit/Dynamo \\
\hline & Rule execution & $\begin{array}{l}\text { Apply the rules (in computer code) to } \\
\text { the extracted BIM-model information }\end{array}$ & Dynamo \\
\hline & Reporting checking results & $\begin{array}{l}\text { Generate a report of the results from } \\
\text { the execution }\end{array}$ & Dynamo \\
\hline \multirow[t]{5}{*}{ Flexibility } & $\begin{array}{l}\text { The separation between the } \\
\text { logic and the data objects. }\end{array}$ & $\begin{array}{l}\text { Separation between data objects and } \\
\text { logic }\end{array}$ & Dynamo \\
\hline & $\begin{array}{l}\text { The separation between run- } \\
\text { and build-time }\end{array}$ & $\begin{array}{l}\text { Separation between the run-time and } \\
\text { build-time users }\end{array}$ & Dynamo \\
\hline & Loosely specified processes & Loosely specified processes & Dynamo \\
\hline & Exceptions & Exception handling & Dynamo \\
\hline & Performance analysis & Monitoring TLP score & Dynamo/Tableau \\
\hline Transparency & BMC processing transparency & $\begin{array}{l}\text { A structured visual representation of } \\
\text { the automated processes }\end{array}$ & Dynamo \\
\hline
\end{tabular}

\subsubsection{Structure of the information processes}

The use of visual programming can be problematic due to the likely complexity of the comprehensive presentation of the code that makes it difficult to users to comprehend. Because of that, visual programming requires sound management of how the processes are structured and presented (Reinhardt and Matthews 2017). The processes are structured according to the hierarchical structure illustrated in FIG. 1. The logic/data objects have been separated into individual nodes and as run- and build-time components. The separation of the run- and build-time users allows a better possibility to maintain the checking operations. The run-time user can alter operations and accommodate aspects envisioned by the BMC system developers or the build-time user. As an example, rulesets often contain measurements like room height without being specific about how such measurements are to be used in all possible scenarios due to practical considerations. Instead, it is the discretion of the users of the rules to provide details of how the rule is interpreted in the specific scenario. If room height is used to specify the flexibility of use of rooms, i.e., can a room be used as an office or a showroom, it is the room height clearance. However, is such clearance constrained by, e.g., lamps or HVAC equipment? Allowing the separation of both run- and buildtime users allows aspects of the operations to be changed in order to make such decisions.

In the prototype, the separation will happen based on the operations (i.e., nodes) that are available for modification by the run-time. Nodes available for project-based adaptations will be denoted as P-nodes. For example, a P-node contains an operation regarding the retrieval of room-height information from a BIM-model. Operations that contain aspects that are important for a company are denoted as C-nodes and cannot be altered by the run-time user. An example of such a scenario is, e.g., when an operation is considered well-structured and highly repetitive. If the calculation of a score related to the building code is explicitly formulated, then it makes no sense to allow the alteration of this calculation by the run-time user. However, in some cases, companies find it important to exceed the minimum of such scores, and therefore it is a company-specific operation that specifies when a score is acceptable according to their quality requirements.

In FIG. 3, a use-case diagram is shown to illustrate the run-time and build-time users' interaction with the prototype. The run-time user authors the BIM-model and uses either the Dynamo player or the Dynamo standalone software to execute operations, while having the possibility to both scrutinize the operations and adapt the P-nodes in relation to the project-based requirements. The build-time user is responsible for managing the scripts in Dynamo that contain the nodes and the visualization of the results in Tableau. He aims to harmonize the scripts used by the run-time user to be as efficient as possible and aligned with the company's strategies and, e.g., updates to the rules. 


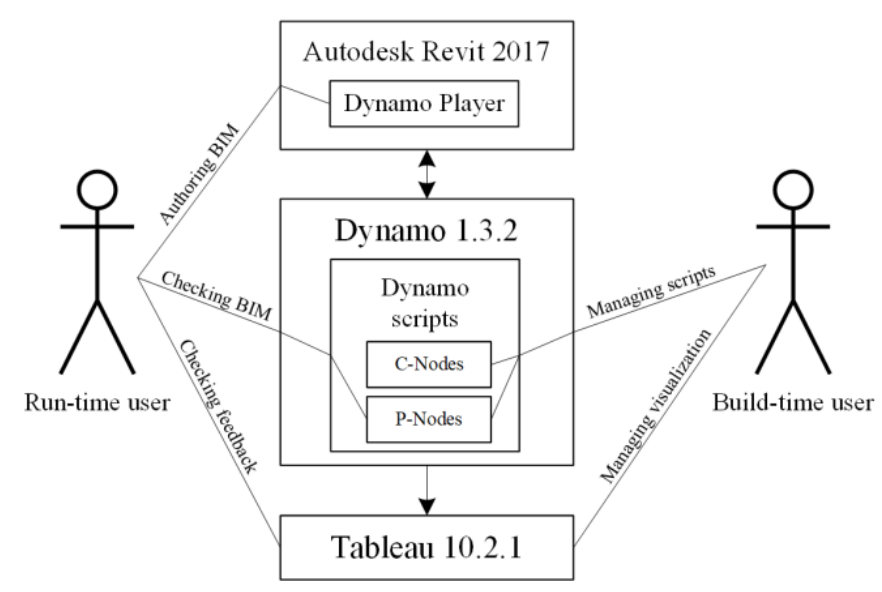

FIG. 3: Prototype use-case diagram

\subsection{Prototype test}

The testing was conducted using rules translated from a criterion from the Danish sustainability assessment method Deutsche Gesellschaft für Nachhaltiges Bauen (DGNB-DK) “office buildings” 2014, 1.1. (Green Building Council Denmark 2014) and a BIM-model of an office building. DGNB-DK contains 40 criteria to assess the sustainability performance of buildings. The third most impactful criterion responsible for $7.5 \%$ of the total score is related to the flexibility and adaptability of the building and is named ECO2.1 Flexibility and adaptability. This criterion has seven sub-criteria, and each sub-criterion can achieve a varying amount of checklist points (TLP) that in total for the criterion can range between 0-100.

The criterion was translated from natural language into the visual programming code of Dynamo, in order to match the functional requirements specified in TABLE 1, including a separation of the data objects and logic, specifying $\mathrm{C}$ - or P- nodes, loose formulation and inclusion of planned exceptions in the process. Moreover, the translation was structured after the hierarchy of nodes as shown in FIG. 1. Because the translation of the rules is not the primary focus of the article, a brief example of how the sub-criterion ECO2.1-1 was translated is presented below (Green Building Council Denmark 2014, p. 143). The four operations were separated into both P- and C-nodes. The P-nodes specify the operations that are editable by the run-time user and the C-nodes specify what is editable by the run-time user only. In this example, decision and action operations are specified as $\mathrm{C}$-nodes because it is defined that the decision must be specified by the run-time user.
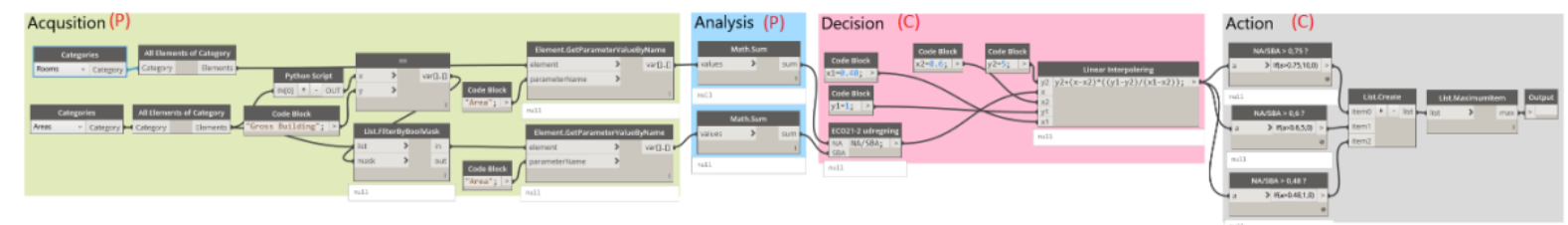

FIG. 4: A view of the contents of sub-criterion ECO2.1-1 separated into operations related to Acquisition (green), Analysis (blue), Decision (pink) and Action (grey). $P$ - and $C$-nodes are marked in red text next to the operation name.

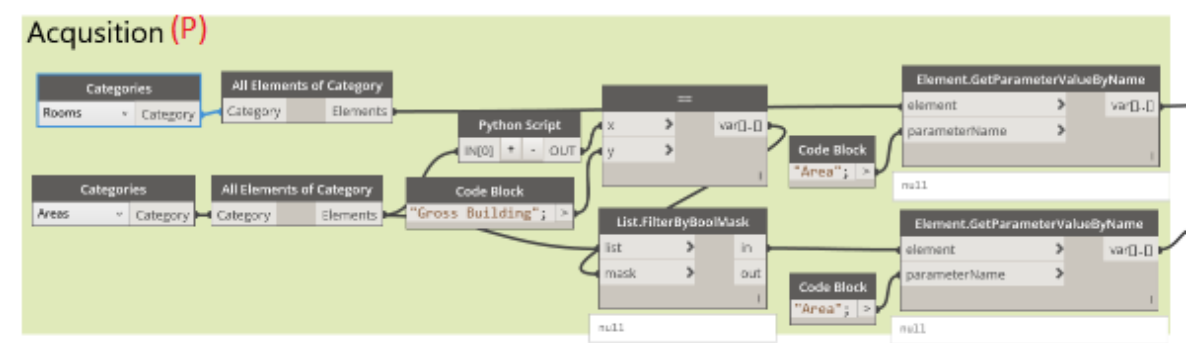

FIG. 5: The Acquisition (green P-node) gathers information related to All Elements of the categories of Rooms and Areas from the BIM-model. 


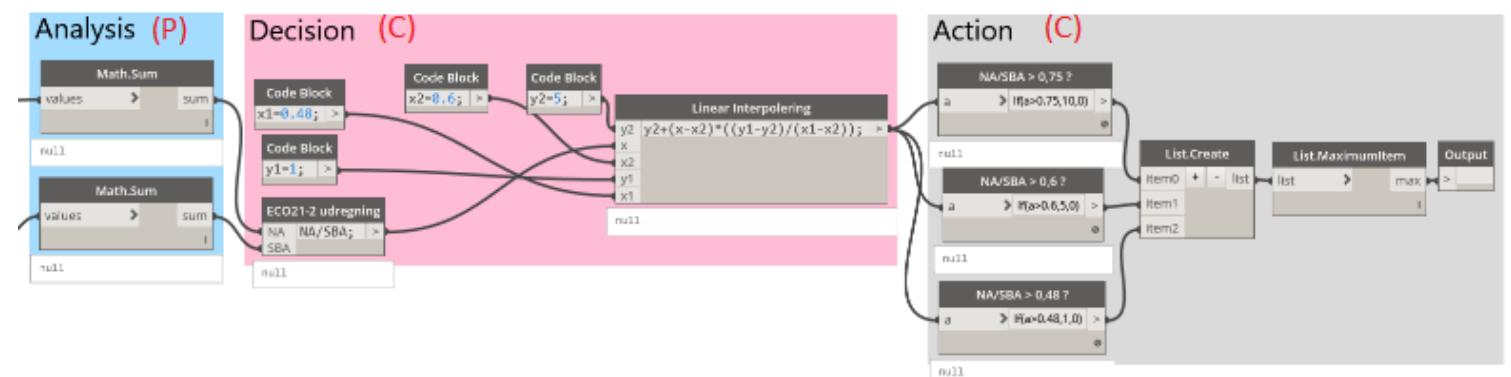

FIG. 6: The specific nodes and wires of the Analysis (blue P-node), Decision (pink C-node) and Action (grey Cnode) operation.

\subsubsection{Assessment of a two-storey office building}

The test was conducted at the users' respective companies. Here a computer was set up with the prototype running and with the test building loaded in Autodesk Revit. The test building (see FIG. 6) represents a two-storey office building in Denmark. The gross building area is $1128 \mathrm{~m}^{2}$, and the building contains a large hall that separates sections in three directions. The building contains 29 different rooms with varying room heights from $2800 \mathrm{~mm}$ to $7100 \mathrm{~mm}$. According to the Danish standards of information levels (Cuneco 2014) the building is specified as information level 3. The building was created for testing purposes, deliberately creating obstructions, e.g., varying room heights.

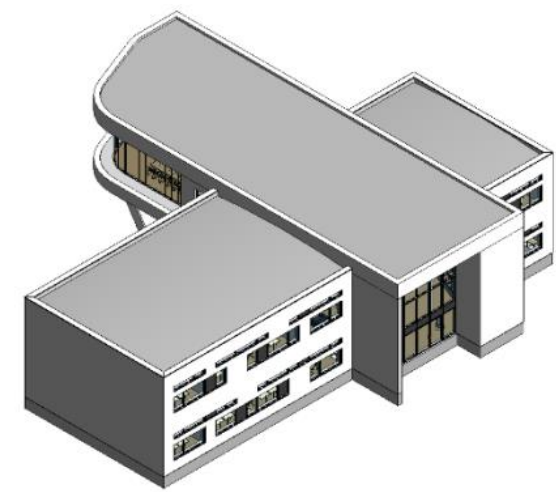

FIG. 6: The two-storey office building optimized for user testing with the prototype

\subsubsection{Participants}

Nielsen (2000) suggests that 5 is the optimal number of users for usability testing. However, if either the domain is complex and comprehensive, or the users' background is diverse, more users could be included. Due to the difference in the backgrounds of the users for this test, we have expanded the number from 5 to 8 . The users were selected based on their professional experience with DGNB, BIM, and BMC, and from a range of small and medium-sized construction industry consultancy companies in Denmark. TABLE 2 shows the participants participating in the user testing.

TABLE 2: Participants in the user testing and interviews

\begin{tabular}{|l|l|l|l|l|l|l|}
\hline $\begin{array}{l}\text { User } \\
\text { nr. }\end{array}$ & Education & Role & Company and size & $\begin{array}{l}\text { BIM } \\
\text { exp. }\end{array}$ & $\begin{array}{l}\text { BMC } \\
\text { exp. }\end{array}$ & $\begin{array}{l}\text { DGNB } \\
\text { exp. }\end{array}$ \\
\hline 1 & $\begin{array}{l}\text { M.Sc. in Construction Management } \\
\text { BIM Manager }\end{array}$ & $\begin{array}{l}\text { Architectural Consulting } \\
\text { Company 1- Medium }\end{array}$ & $\begin{array}{l}15 \\
\text { years }\end{array}$ & $\begin{array}{l}0 \\
\text { years }\end{array}$ & 0 years \\
\hline 2 & $\begin{array}{l}\text { B.Sc. in Architectural Technology and } \\
\text { Construction Management }\end{array}$ & BIM Developer & $\begin{array}{l}\text { Architectural Consulting } \\
\text { Company 1- Medium }\end{array}$ & $\begin{array}{l}2.5 \\
\text { years }\end{array}$ & $\begin{array}{l}2 \\
\text { years }\end{array}$ & 0 years \\
\hline
\end{tabular}




\begin{tabular}{|l|l|l|l|l|l|l|}
\hline $\begin{array}{l}\text { User } \\
\text { nr. }\end{array}$ & Education & Role & Company and size & $\begin{array}{l}\text { BIM } \\
\text { exp. }\end{array}$ & $\begin{array}{l}\text { BMC } \\
\text { exp. }\end{array}$ & $\begin{array}{l}\text { DGNB } \\
\text { exp. }\end{array}$ \\
\hline 3 & $\begin{array}{l}\text { Cand. Scient. Techn. in Building } \\
\text { Informatics }\end{array}$ & $\begin{array}{l}\text { BIM Process } \\
\text { Developer }\end{array}$ & $\begin{array}{l}\text { Engineering Consulting } \\
\text { Company 1 - Medium }\end{array}$ & $\begin{array}{l}3 \\
\text { years }\end{array}$ & $\begin{array}{l}2 \\
\text { years }\end{array}$ & 0 years \\
\hline 4 & $\begin{array}{l}\text { B.Sc. in Architectural Technology and } \\
\text { Construction Management }\end{array}$ & DGNB Auditor & $\begin{array}{l}\text { Architectural Consulting } \\
\text { Company 2 - Medium }\end{array}$ & $\begin{array}{l}7 \\
\text { years }\end{array}$ & $\begin{array}{l}0 \\
\text { years }\end{array}$ & 3 years \\
\hline 5 & $\begin{array}{l}\text { B.Sc. in Architectural Technology and } \\
\text { Construction Management }\end{array}$ & $\begin{array}{l}\text { BIM Manager \& } \\
\text { DGNB Consultant }\end{array}$ & $\begin{array}{l}\text { Engineering Consulting } \\
\text { Company 2 - Medium }\end{array}$ & $\begin{array}{l}3 \\
\text { years }\end{array}$ & $\begin{array}{l}0 \\
\text { years }\end{array}$ & 3 years \\
\hline 6 & $\begin{array}{l}\text { B.Eng } \\
\text { Engineering Consulting } \\
\text { Company 3 - Small }\end{array}$ & $\begin{array}{l}0 \\
\text { years }\end{array}$ & $\begin{array}{l}0 \\
\text { years }\end{array}$ & 2 years \\
\hline 7 & $\begin{array}{l}\text { B.Sc. in Architectural Technology and } \\
\text { Construction Management }\end{array}$ & BIM Manager & $\begin{array}{l}\text { Engineering Consulting } \\
\text { Company 3 - Small }\end{array}$ & $\begin{array}{l}4 \\
\text { years }\end{array}$ & $\begin{array}{l}0 \\
\text { years }\end{array}$ & 0 years \\
\hline 8 & $\begin{array}{l}\text { B.Sc. in Architectural Technology and } \\
\text { Construction Management }\end{array}$ & $\begin{array}{l}\text { BIM Manager \& } \\
\text { DGNB Consultant }\end{array}$ & $\begin{array}{l}\text { Engineering Consulting } \\
\text { Company 4 - Small }\end{array}$ & $\begin{array}{l}3 \\
\text { years }\end{array}$ & 1 year & 1 year \\
\hline
\end{tabular}

\subsubsection{Data collection and analysis}

The data from the process and results are obtained from three sources: semi-structured interviews, video observations, and performance metrics. Triangulation is used to ensure confidence in the tests, and includes gathering data from various sources to establish confidence (Jick, 1979; Kaplan and Duchon, 1988). The data are analyzed by categorizing using affinity diagramming (Beyer and Holtzblatt 1997). The video observation was conducted to assess how the users interacted with the prototype during the automated assessment, and how they assessed the building unaided without the support of the prototype. The interviews were conducted to enquire about how they perceived the consistency, precision, flexibility, and transparency and trust of the prototype. Quantitative metrics were gathered to assess the speed and precision of the ECO2.1 assessment. See TABLE 3 for an overview.

TABLE 3: Overview of metrics used to evaluate the prototype

\begin{tabular}{|l|l|l|l|}
\hline Topic & Description & Quantitative metrics & Qualitative metrics \\
\hline Speed & $\begin{array}{l}\text { Comparing time used for } \\
\text { assessment }\end{array}$ & $\begin{array}{l}\text { Time deviation } \\
\text { percentage between } \\
\text { traditional and } \\
\text { prototype test case }\end{array}$ & Not relevant \\
\hline Consistency & $\begin{array}{l}\text { Comparing assessment } \\
\text { results from the assessments }\end{array}$ & $\begin{array}{l}\text { Score deviation } \\
\text { between results }\end{array}$ & $\begin{array}{l}\text { How the users experienced the } \\
\text { quality of consistency }\end{array}$ \\
\hline Flexibility & $\begin{array}{l}\text { The ability of the user to } \\
\text { adjust to changes in DGNB } \\
\text { and the BIM-model }\end{array}$ & Not relevant & $\begin{array}{l}\text { How the users experienced the } \\
\text { ability to be flexible to unforeseen } \\
\text { changes in DGNB and the BIM- } \\
\text { model }\end{array}$ \\
\hline $\begin{array}{l}\text { Transparency } \\
\text { and trust }\end{array}$ & $\begin{array}{l}\text { The ability of the user to } \\
\text { understand and trust BMC } \\
\text { processing }\end{array}$ & Not relevant & $\begin{array}{l}\text { How the users experienced the } \\
\text { transparency and trust of each } \\
\text { case }\end{array}$ \\
\hline
\end{tabular}

According to Jewitt (2012), video recordings can support exploratory research and are a suitable complementary source of data. Video observations can contribute to how users interact with technology in a context (Nielsen and Kaufmann 1993). In this study, a total of 8 hours of video recordings were gathered. The semi-structured interviews were conducted to allow poor responses to be overcome, as well as further exploration of values and 
attitudes, and the opportunity to evaluate the validity of the respondents' answers (Barriball and While 1994). The questions were devised based on the theory presented earlier in relation to flexibility (Reichert and Weber 2012), and transparency and trust (Inagaki et al. 1998, Moray et al. 2000, Hoffman et al. 2013). The quantitative metrics were derived from Fish's (2012) theories of knowledge automation used to assess the quality of automation quantitatively. We compared the time taken for the traditional assessment of the building with the time taken for the automated assessment.

\section{RESULTS FROM THE TESTS}

In the following sections will we unfold both the qualitative and quantitative results.

\subsection{Quantitative results}

The quantitative results showed that the users spent an average of 29 minutes assessing the performance of the building unaided according to ECO2.1. The users' and the rule developers' total scores differed, with total scores ranging from 25 to 50. However, the results from criterion ECO2.1-2 were consistent, as shown in TABLE 3. The rest of the users' sub-criteria scores varied and were different from those of the rule developers. User 5 did not find that the BIM-model contained enough information to assess sub-criteria 4 and 6.

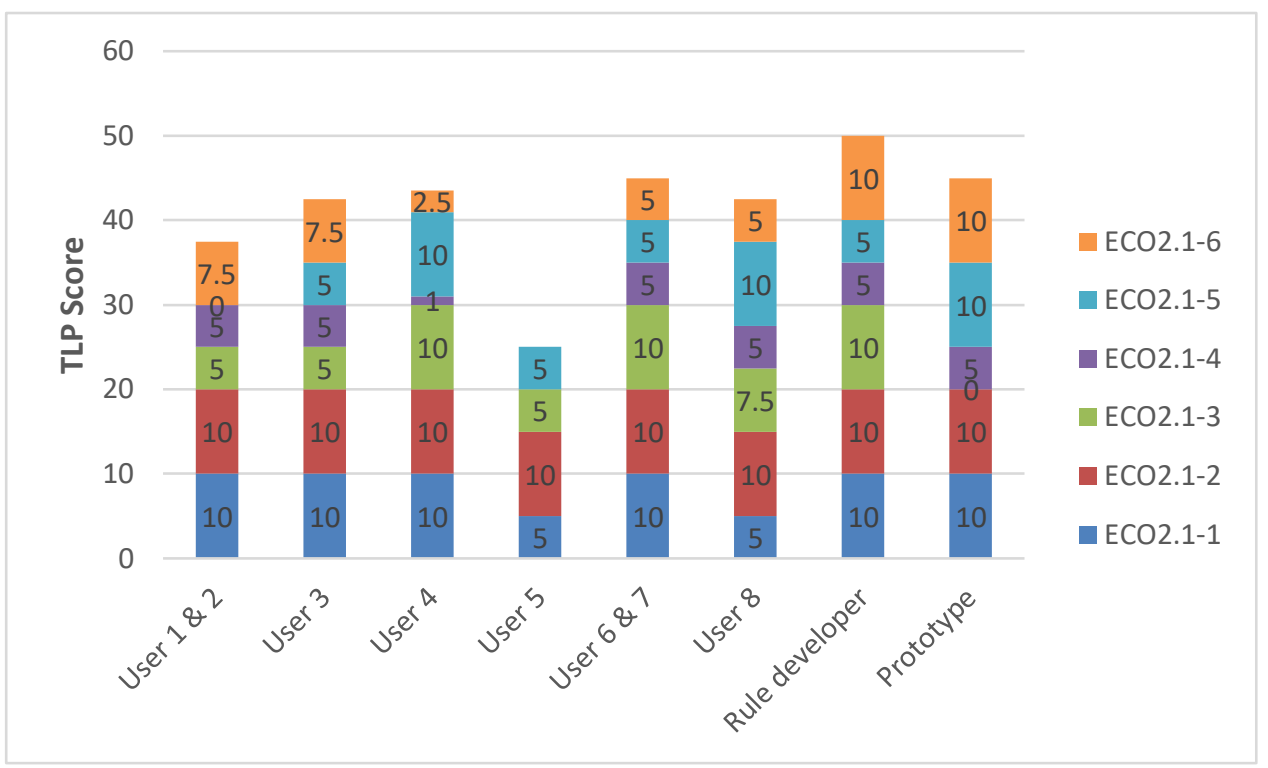

FIG. 7: Results from the unaided assessment of ECO2.1

The score results of the unaided assessed criteria were on average $15 \%$ lower than those of the rule developer. Only sub-criterion ECO2.1-2 matched the rule developer's score. ECO2.1-5 was on average $14 \%$ higher than the rule developer's score, and the remaining sub-criteria scored lower. The prototype assessed the building within one minute, and the average score was $10 \%$ lower than the rule developers. The individual sub-criteria scores from the prototype show that two sub-criteria differ from the rule developer: ECO2.1-3 and ECO2.1-5. The results are shown in TABLE 4. The results also indicate that users with DGNB experience scored lower (37.8 TLP points on average) than users without DGNB experience (40 TLP points on average).

TABLE 4: Results of the traditional and prototype assessments.

\begin{tabular}{|l|l|l|}
\hline Metric & $\begin{array}{l}\text { Traditional assessment } \\
\text { results (result on average) }\end{array}$ & Prototype results \\
\hline Speed & 29 minutes & $<1$ minute \\
\hline Precision ECO2.1 & $85 \%$ & $90 \%$ \\
\hline Precision ECO2.1-1 & $83 \%$ & $100 \%$ \\
\hline
\end{tabular}




\begin{tabular}{|l|l|l|}
\hline Metric & $\begin{array}{l}\text { Traditional assessment } \\
\text { results (result on average) }\end{array}$ & Prototype results \\
\hline Precision ECO2.1-2 & $100 \%$ & $100 \%$ \\
\hline Precision ECO2.1-3 & $71 \%$ & $0 \%$ \\
\hline Precision ECO2.1-4 & $84 \%$ & $100 \%$ \\
\hline Precision ECO2.1-5 & $114 \%$ & $150 \%$ \\
\hline Precision ECO2.1-6 & $55 \%$ & $100 \%$ \\
\hline
\end{tabular}

\subsection{Interviews and observations}

This section presents the results from the interviews and observations conducted with the users from the design practice. The first sub-section presents the results from the traditional assessment of the building. The next subsection presents the results from the users using the prototype to assess the building.

\subsubsection{Traditional assessment}

The users had different approaches on how they used the BIM information for assessing the criteria. Their primary approach to gathering information was by creating schedules in Revit with quantities for ECO2.1. Others made use of the building's 3D visualization to assess specific sub-criteria, for example assessing ECO2.1-4 vertical access. The differences between the users' results were mainly due to the different methods of assessing the building. An example of this difference was observed when the users assessed the ECO2.1-2 sub-criterion. In the description of ECO2.1-2, it was specified that the room height had to be measured from the top raw floor to the bottom raw ceiling. The definition "raw floor" is not a standardized term in Danish construction vocabulary but has connotations related to unfinished surfaces. This vague wording allowed the interviewees to define terms differently, such as, e.g., the raw top floor as the top finish of the floor, as the top core structure (such as a concrete slab), or as a top screed. Though this was a source of inconsistency, the interviewees argued that the vague wording allowed interpretations enabling the rules to be applied to the projects better while retaining the intent of the rules. In one example, an interviewee argued that the definition of the primary or secondary use of a room regarding subcriterion ECO2.1-2 is open to interpretation. "In a discussion between $D K-G B C$ and me, in relation to a kindergarten, I made the argument that the lobby was a primary room because it had a pedagogical function for the children in relation to waving goodbye to their parents." (User 5). Not only did the vaguely formulated rules allow the users to use their discretion, but they also served as a competitive factor. The same interviewee had experienced that a collaborator even misused the interpretations of DGNB to provide a better competitive factor. "The interpretation of the criteria is a competitive factor. For example, a contractor asked if we could exempt the calculation of thermal bridges and linear losses in the energy calculation and only focus on u-values." (User 5).
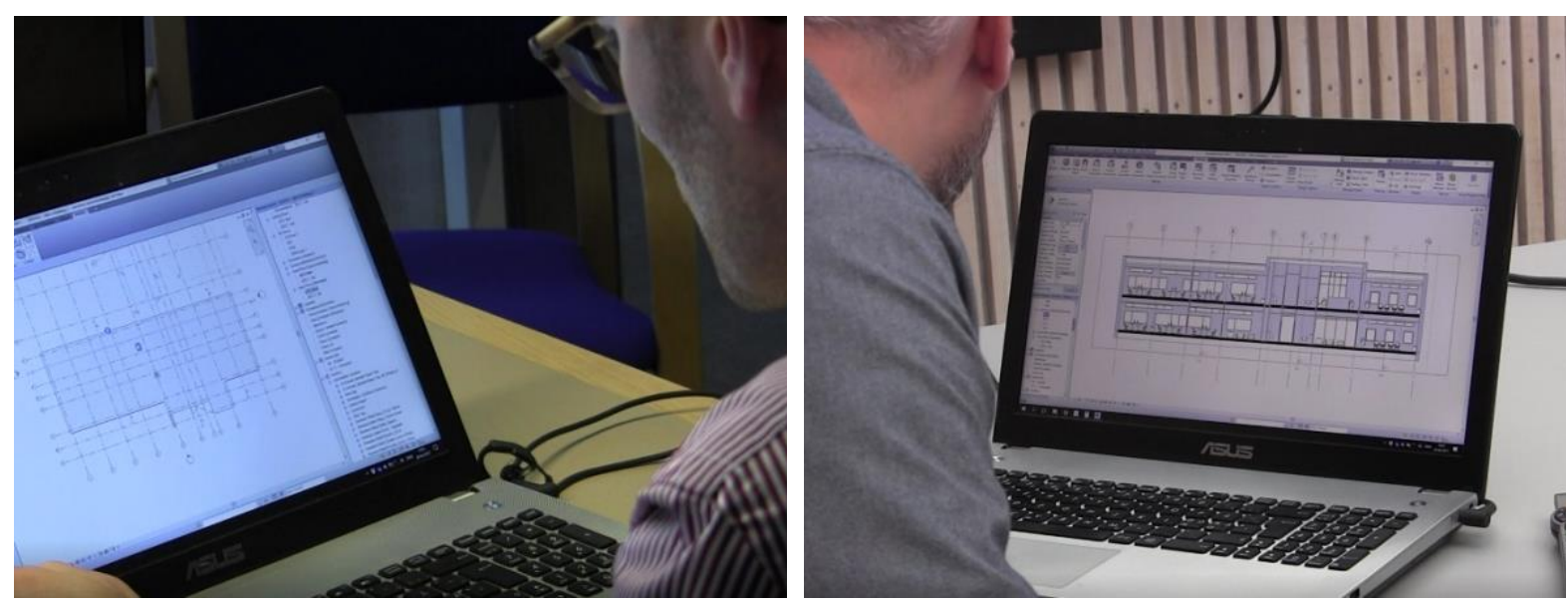

FIG. 8: Screenshots from the video observations showing two users assessing the building unaided according to ECO2.1 
The traditional assessment was conducted, and the users were able to assess the score of the building. The results from the traditional assessment showed that even the users with DGNB-DK experience applied different methods that led to different results. The observations showed that many of the users trusted the quantities seen from the sections in Revit's representation of the BIM-model. However, some of the rooms were not visible from the sections and would be left out of the user's traditional assessment, which affected their calculation of the score.

\subsection{Using the prototype to assess ECO2.1}

The interviewees expressed the opinion that the use of the prototype would support their practice and ease their workload related to assessing a building's performance according to DGNB. The interviewees agreed that the prototype could lead to increased consistency of the assessment due to the explication of the many vaguely formulated rules. Moreover, it would reduce the time, repetitions and effort spent on assessing the performance of the building, which could result in more regular assessments throughout the design process.

In all the interviewees' companies, Dynamo is increasingly used. However, Dynamo was considered a BIM specialist system that would be difficult for non-BIM specialists (e.g., engineers) to use. Moreover, Dynamo was perceived as being less stable than other software solutions. "It is sensitive to wrong inputs; for example, if you were to use a symbol instead of a number it would crash completely." (User 1). One company that already used Dynamo to automate some aspects of their work argued that they consider using Dynamo to be better for tasks at hand that need automation, rather than tasks that require high certainty. "Dynamo is a quick and dirty system that users can apply to automate tasks at hand." (User 1). Another interviewee argued that "There is a certain degree of uncertainty associated with the user interface with Dynamo." (User 3).

\subsection{Transparency}

The interviewees all agreed that a key benefit of using the prototype was its transparency. "It is easy to understand, and it is pedagogically expressed." (User 4). However, the interviewees stated that the prototype had to be tested by company specialists to review the BMC processing. The interviewees would use the prototype if they could review the processes and test it on various projects. One interviewee argued that the main uncertainty is the BIMmodel.

One interviewee argued that, through the explicit management of the assessment process with the rules, it would allow for a more reflective interpretation for the company. The traditional assessment of a building is a highly individual process, making it difficult to share experiences among colleagues. The interviewees argued that the explicit and visual formulation of the prototypes processes allowed individuals to make their processes clear. This allows a clearer expression of how a score is achieved. If the designers authoring the BIM-model are aware of how their design choices affect the score of the assessment, they can design better for assessment performance. The interviewees argued that designers working on a building design (e.g., the BIM modellers) typically do not have much insight on the rules constraining the design. The prototype can assist designers to better understand the consequences of their design choices during the design, instead of later in the process when evaluated by, e.g., a DGNB consultant or auditor. "It especially gives me a better platform for communication to allow the participants to see things in a context; this is done better in the prototype." (User 6). Not only does it communicate the processes of the assessment better, but it also highlights the information needed in the BIM-model to conduct such an assessment. A lack of information in the BIM-model is also an indicator of decisions that need to be made in the project. The prototype would be able to highlight such needs through notifications but would require that it be made transparent; otherwise, information missing from the BIM-model can result in the prototype calculating flawed results.

One interviewee argued that having more explicit information requirements by using a system like the BMC prototype would better highlight the exchange of information needed in a project. Also, it would specify what information is missing, increasing awareness of what information is needed and why. This is something that is largely missing at present. "The use of a prototype like this would increase the quality of information in the BIMmodel. In many cases, we receive information from the architect, which results in missing information in relation to our needs." (User 7). Another interviewee argued "If information like the primary or secondary use parameter in rooms is missing, the prototype makes the designers notice that." 


\subsubsection{The balance of accountability between the build- and run-time users}

The interviewees argued that black-boxing, in general, was undesirable. It could be necessary to restrict and standardize some aspects of BMC processing to ensure consistency and validity. Most of the interviewees argued against black-boxing. They suggested better control of certain nodes in the prototype with the ability to either lock or lock with the possibility of override certain nodes and processes. However, one interviewee argued that "For me, it was OK if everything was black-boxed in relation to if someone says that is $O K$, as long as the responsibility does not lie with me." (User 5). This comment was directed at whether it was possible for him to completely trust the prototype by having it, e.g., certified by DK-GBC, so that he did not need to establish trust beyond that. The rest of the interviewees argued that there was a need to be able to manipulate the BMC-prototypes process to adjust to the needs of the projects and disagreed with the black-box approach. However, they expressed that it would be beneficial if there were more control of central nodes in the process. "The prototype could benefit from a more central place to set up the rules." (User 3). One argued that it could improve maintenance to control the processes centrally, to update and lock the nodes better than with Dynamo in the prototype.

One interviewee (User 1) stated that it was of great importance to consider the balancing of benefits and trade-offs in accountability between the user and the organization. At both levels, the individual and the organization are accountable for the work they carry out, and at both levels, they have different objectives for when the system either constrains or benefits the projects. The interviewees added that it is the responsibility of the run-time user to manage a project's budget and the time to decide when, and if, to use a system. Understanding the BMC process is vital to assess if it would be of benefit in a certain situation. However, the interviewees explained that only a few people would make an effort to understand the BMC system completely. "90\% of people are not interested in how things are executed in detail; it is only "the nerds" that would go into detail... The rest do not care about following the information from A to B." (User 1). The interviewees argued that it would still be necessary to have manual validations by domain specialists of the automated processes to ensure that the automation is valid and/or for DK-GBC to validate the system.

Other interviewees argued that for specialists like engineers to use the prototype, it would require them either to be fully able to scrutinize how the information is processed or to trust the developer or organization that is responsible for its implementation. "In general, $70 \%$ of all engineers would ask questions on how the prototype works and try to understand the smallest detail." (User 5). The interviewees further added that it is considered essential that the users are in control. "It is important for the users of the prototype to identify when the prototype automates critical tasks where the user is responsible for how it is processed and when it just gives a direction of the performance." (User 1). This evaluation of the prototype was considered necessary to ensure certainty in the processes and for the user to decide if the quality of the automation adequately supports his/her work.

\subsubsection{Understanding the limitations}

The interviewees had previously experienced challenges with overtrust and argued that using the prototype would still require a certain level of domain-specific knowledge. The use of the prototype requires that the user can review the BMC processing and react to issues. The decoupling between the user's domain knowledge and the BMC processing can potentially lead to dangers associated with such automation. In the current practice, there have been examples of overtrust, and these had to be considered as a significant threat when integrating systems which automate a design practitioner's work. "We created a prototype to calculate daylight, where we and the software did not communicate its limitations enough. This resulted in a user taking screenshots as documentation which was sent to the architect." (User 5).

The interviewees expressed that the prototype allowed the user to understand its limitations because of the transparency of the processes to a certain degree. However, there is a need to communicate the limitations to the users further. One interviewee suggested that more notifications on the functionalities would help communicate the limitations of the prototype, for example, when the BMC processing is for purposes that require high certainty. "It is important to make the users aware that it is only a simulation, what you get out is not the exact truth but a simulation, but to direct the design towards optimization. You have to be able to interpret this with your professionalism." (User 1). Another interviewee argued that the prototype needed notifications when the performance of the building was close to achieving a point. "If the calculation is close to achieving points or more points, there should be a notification from the prototype. For example, if the width of a building is 14.7 and not 14.5 that allows for points." (User 6). 


\subsubsection{Project and organizational learning for continuous improvement}

One interviewee argued that the use of the prototype would allow tracking of the evolution of the different BIMmodels' performance, which would be difficult to achieve in a traditional approach. "The use of the prototype is an advantage because it can spot issues that are difficult for me to find. It makes the user aware of underperforming aspects of the building design, which need action. For example, if the architect delivered a new model and the performance decreased in aspects of DGNB, I would be able efficiently to locate the issues and assess the causes and actions to counter the issues." (User 5). Besides the score itself, one interviewee argued that the benefit was based on the user's ability to understand how the score was achieved. As an example, when a performance score is low in one criterion it provides the user with rationale for that score, e.g., that rooms in a certain area have a low room height.

The interviewees argued that the prototype needed functionality to better express the BMC-prototypes' performance history in terms of how both the BIM-models and assessments perform. "It would be nice if it was possible to track the DGNB performance history, which would allow us to identify reasons for the performance changes. We can use that for documentation both inside and outside the company. The way we do it now, we have difficulties gaining a historical perspective of how and what made our buildings perform." (User 4). Visualizing the performance throughout the design process would enable the user and the organization to learn how the different design choices affect the performance. This was an important issue for the interviewees, who also suggested that more notification was needed to identify aspects of the sub-criteria that could be improved with little effort but with a high impact on the performance. "In some cases, I would have been able to achieve points with minor changes to the design, and the prototype does not notify me when this happens." (User 6).

\subsection{Flexibility}

The interviewees argued that the prototype would provide users with the flexibility to interpret the criteria in the contexts of their projects as well as of the company. Though the prototype provided functionalities of flexibility, it raised concerns for one interviewee about handling the varying quality of the information of the BIM-model. "It is a dangerous assumption for a system like a prototype to rely on a complete set of BIM-information. As an assessor, we often receive BIM-models we did not make ourselves. This can be a problem if there are inconsistencies in the BIM-model that lead to issues in the prototypes processing." (User 6).

Design information evolves and the quality of the information from the BIM-model is often not of a high enough quality until the end of the project, when most of the decisions of the project have already been made. One example explained by an interviewee was: "It can be a problem for the designers to properly classify objects in the BIMmodel, which contain some uncertainty." (User 3). Another interviewee argued that even if they attempted to specify the information needs, it would often not comply with these requirements. "We see many examples in our work where we receive BIM-models of varying quality. Sometimes we get BIM-models where everything is created in generic models because it was easier for the BIM-modeller." (User 3). Reasons for this were argued to be related to the often diverging goals of modelling. "The reason why the quality of the BIM-model varies is that it is used to generate drawings." (User 3). Therefore, the interviewees argued that the BMC system must support the dynamic process of design better, which requires that the prototype must be able to use the information better at the various stages of maturity and quality.

However, people are often from different companies, as well as having different goals and responsibilities. Requiring a designer to create a BIM-model for another company's benefit, and a poor understanding of other designers' needs results in poor quality information, emphasizing the need for transparency of the processes. One interviewee expressed: "There is a danger that the person who uses the prototype does not know the context of how the information is processed, which would be a source of uncertainty." Previous attempts to counter this issue have been by specifying the information needs in detail. However, the interviewees argued that there were still embedded challenges in the use of systems using such information because designers from collaborating companies either put little effort into providing good quality information or still lacked a complete understanding of how the information is intended to be used. "We prefer the BIM-model to contain DGNB specific parameters, like for rooms, specifying if they are for secondary or primary use." (User 7) - referring to the ability to specify the role of the rooms ad hoc. 
The interviewees expressed that the prototype allowed them to be flexible, both regarding BIM-models of varying quality, and relating to the specific processes of the project. "The more simply the processes are formulated the better, because it allows the users to adapt to the changing building design" (User 4). The interviewees argued that the approach to formulating the processes loosely would improve their understanding of the processes themselves, but also better enable them to adapt the processes to their project contexts. "The prototype allows adapting to the real-world processes because of the many changes in, for example, the criteria." (User 4). Moreover, the looseness of the formulated sub-criteria was experienced as a good approach from a cost-value perspective because it would lessen the resources otherwise required to create and maintain highly specific and detailed processes. "It is important that the automated processes are loosely defined because the effort and complexity of specifying the automated processes require too many resources."

Many of the interviewees argued that it was critical to ensure control of the maintenance of the rules. "In other software, we are challenged by updates to the processes that in some cases significantly modify the results." (User 6). The versioning of the processes in the prototype had to be managed either through the custom nodes or through manual handling of the Dynamo scripts.

\section{DISCUSSION}

The prototype made use of modularisation to enable the user to more easily understand and adapt the processes according to the context and reduce the visual complexity of VPL in Dynamo. The modularisation was reported to be helpful for the interviewees to understand how the information was processed, allowing them to achieve a simplified overview. However, there are limitations in the available functionalities of Dynamo because its use is not directed towards model checking. It is possible to create custom nodes in Dynamo that are locked. However, efficiently managing how these nodes are maintained was reported as being sub-par. An interviewee argued that the use of Dynamo was a hazard for the company because it allowed too much alteration of the processes by the run-time users. Further control and separation seem to be required between the run-time users of BMC systems and the processes that the build-time users employed, as indicated by the interviewees. However, as Reinhardt and Matthews (2017) note, the use of visual programming can be an efficient method of conducting BMC through good management. But as Cavalliere et al. (2019) argues, that using VPL-based solutions require strict enrichment and management of the information in the BIM-models.

Whitley (1996) argues that the main challenge of using VPL is "Given the range of information required in programming, can a VPL highlight enough of the important information to be of practical benefit?". The interviewees were able to scrutinize how the information was processed, even by users who had limited experience with BIM. A lack of looseness can result in comprehensive and complex processes that can potentially overburden the user with information that is not important and lead to rejection, as noted by the interviewees. The prototype was judged to require a better interface to communicate various notifications. For example, there needs to be an indication of when the performance of a building is close to obtaining points but does not comply. Dynamo does not provide many opportunities to control and constrain the run-time user or allow control functionalities of the build-time user compared to other systems like business process management systems (Koncevics et al. 2017). However, currently no business process management solutions exist specifically for use in the construction industry.

The interviewees' TLP scores for the sub-criterion ECO2.1-3 were significantly different from the score of the rule developer and the prototype. The reason for this difference could be identified as the literal interpretation of the term depth. The sub-criterion is assessed based on the depth of the building. To understand this measurement, you have to understand its context, and the flexibility of the building's uses. This means that it is up to the individual assessor to subjectively formulate what that means and how it affects the measurement of the building depth. While standards can provide a uniform way of specifying the depth of a building, many scenarios can occur where strictly formulated rules neglect unique aspects of the building and cause potential issues. In our case, the rule was translated literally from the specifications stated in the DGNB-DK into the prototype, yet the different interpretations resulted in different results in the traditional assessment test.

The rule specifies that it is the complete depth of the building measured from outer wall to outer wall. Merriam Webster defines depth as "the direct linear measurement from front to back." The walls from the BIM-model were then transformed into 2D lines in a plan view, where the depth was measured as a direct linear measurement conducted from the front to the back. The front and back were defined as the narrowest points. In comparison with 
the unaided assessed scores, this approach was different. The general approach would be to measure the front and back of the narrow "body" of the building and not the building per se. Since the building is T-shaped, the result was very different from the prototype. This is an example of a criterion that either needs to be very complex in the prototype BMC processing, or provide the user of the prototype with transparent processing so that he can adapt the automation to the specific design where it is used.

The results of the interviews show that traditional methods of assessing buildings allow for a company-based interpretation of rules to both their own and the rule enactor's advantage. The problem with hard-coded and blackboxed BMC systems is that they potentially restrict the users, preventing them from adapting the rules constricting their work of assessing the building. Adopting a BMC system then becomes a balance between the benefits and constraints of automation. The results from the interviews indicated that BMC systems like the prototype must give more consideration to how rules should be interpreted, allowing the users to make adaptations. It has previously been argued that construction projects are highly reliant on contextual knowledge and for that reason often require that the processes can accommodate rapid changes (Demaid and Quintas 2006). For example, the knowledge that is created during the process of designing a unique building could affect how the rules are supposed to be used, such as re-interpreting the meaning of what a primary room is. The integration of rules in the prototype was an attempt to let the designers adapt the use of the rule while still achieving its intent. One of the results, an interviewee's "rule-breaking" in the definition of primary and secondary rooms in the traditional assessment, led to the designer's ability to adapt the rule in the context of its use. The goal of the rules in the criterion is to ensure that the design is flexible in its use. However, letting the users adapt the rules might result in inconsistent assessment and increase the need for manual labour. Additionally, it would be more difficult to control how the users conducted the BMC systems assessments.

Traditionally, it is the users that have the main responsibility for correctly applying the rules and they are required to present documentation on how the rules are interpreted. However, by using contemporary BMC systems, it is the system developers that specify how these rules are interpreted, but these are hard-coded and black-boxed, limiting the user's ability to understand and adapt the automated assessment according to her/his context. However, it is still the assessor who is liable for the correct assessment of the rules, whereas for BMC developers to take on such liability can be problematic. This perspective is aligned with the notions from Preidel \& Borrmann (2015b) who argues that "The correctness and accuracy of a compliance checking is the responsibility of the reviser and cannot be transferred to a machine because of legal reasons." Allowing the users to understand and adapt the rules to their context would better align the responsibility with the users rather than the creators of BMC systems.

As the interviewees commented, unnecessary explication could remove competitive advantage and rule out contextual adaptations that could potentially spark innovative solutions while preserving the intents of the rules. Demaid and Quintas (2006) argue that negligence of context-based knowledge has previously led to problems. They argue that the "holy grail" of converting all knowledge into explicit knowledge had historically failed on many accounts including the failure of many promised expert-systems. In many cases, it is impossible to create rules for BMC systems that consider all possible scenarios because this requires that the developers envision all possible contexts of use. Limiting the user's ability to adapt the rules could lead to unforeseen consequences that could potentially negate the upside of using BMC systems.

\section{CONCLUSION \& FURTHER RESEARCH}

In this article, we aimed to provide insights into improving the functional performance of BMC systems that will allow better adaptation of the systems into practice. To gain such insights we applied DSR that emphasized the development and evaluation of artifacts. Therefore, a BMC prototype was developed based on a set of functional requirements aimed to accommodate existing socio-technical challenges, focusing on transparency and flexibility. Moreover, we were able to test the prototype with practitioners and found the prototype to provide functionalities with both the intended transparency and flexibility and reduced time spent on assessing the building design, allowing for more consistency compared to the traditional assessment methods.

The practitioners' use of the prototype allowed us to explore the socio-technical challenges (i.e., transparency and flexibility) holding back the use of BMC systems and the use of considerations to relieve these challenges. The results were discussed, and the interviewees indicated that by allowing them to scrutinize and adjust the processes, they would have more trust in the system. The more trust in the system, the more comfortable they are in using it for critical purposes such as assessing sustainability in a BIM-model. Moreover, it allows users to identify 
shortcomings of the processes in the context of their uses and to adapt them as needed. Equally important, it allows companies to enforce specific company-required behaviour, with the ability to restrict specific nodes in the process. While the prototype enabled the interviewees to assess the BIM-model according to the rules of ECO2.1, there were shortcomings. Further functionalities related to control and maintainability were required to analyze the use of such a prototype, and better handling of notifications to users and a more structured separation between logic and information were considered necessary. In a software system like Dynamo, it is possible to create notifications, separate logic, and information in nodes. However, the interface for managing this was considered possible, but not sufficiently structured. Moreover, the indications about the need for transparency and flexibility identify a dichotomy between black-boxed/hard-coded and transparent/flexible. While complete control over the BMC system might overburden the run-time user, there is a need to communicate the processes in the BMC system and to allow for a certain flexibility. An imbalance in the dichotomy could have unintended consequences for the practices that BMC systems aim to improve. For example, as noted by Refviks et al. (2014), the failure to use BMC systems might be due to the "cost" outweighing the benefits of using them.

While limiting the transparency and flexibility offers easier development and control of the BMC systems, it also lessens the involvement of the users. The lack of involvement reduces the ability to apply the tacit knowledge often required to properly automate processes like assessment. A failure to recognize the need for tacit knowledge has previously been challenging for the expert systems movement. Demaid and Quintas (2006) noted that the main failure for expert systems was the inability to involve the users and thereby embed their tacit knowledge into the systems without formalizing it into rules. When rules are formalized in BMC systems that limit the user's involvement, this can reduce the user's ability to perform using his/her own discretion. Rules were never meant to be too explicit in the first place, but as McLean (2003, p. 23) argues: "Outputs of the political system include laws, rules, judicial decisions, and the like, regarded as the authoritative allocation of values. " Rules in the construction industry are intents of how we want the environment we live in to be, and when rules become formalized there is a risk that the values and intents are set aside by being too inflexible. Rule compliance is then for the sake of the rule. Allowing the users of BMC systems to adjust the interpretation of rules through the ability to understand how they are applied in the system (transparency) and how they can adjust them (flexibility) is one approach to balance the explicitness.

We recommend that further qualitative studies are conducted to better understand the socio-technical characteristics of the use of BMC systems. Further research is needed on how BMC systems can provide a balanced approach to handling rules.

\section{REFERENCES}

Ahmed, S., 2018. Barriers to Implementation of Building Information Modeling (BIM) to the Construction Industry: A Review. Journal of Civil Engineering and Construction, 7 (2), 107.

Amor, R. and Dimyadi, J., 2020. The Promise of Automated Compliance Checking. Developments in the Built Environment, 5 (October 2020), 100039.

Amor, R. and Dimyadi, J., 2021. The promise of automated compliance checking. Developments in the Built Environment, 5, 100039.

Barriball, K.L. and While, a, 1994. Collecting data using a semi-structured interview: a discussion paper. Journal of advanced nursing, 19 (2), 328-335.

Beach, T.H., Hippolyte, J.L., and Rezgui, Y., 2020. Towards the adoption of automated regulatory compliance checking in the built environment. Automation in Construction, 118 (September 2019), 103285.

Bell, J.R., 1973. Threaded code. Communications of the ACM, 16 (6), 370-372.

Bertelsen, S., 2003. Complexity - Construction in a New Perspective. International Group of Lean Construction, 12.

Beyer, H. and Holtzblatt, K., 1997. Contextual design. 1 edition. San Fransisco: Morgan Kaufmann.

Building And Construction Authority, 2015. Code of practice on: Buildability.

Cavalliere, C., Dell'Osso, G.R., Favia, F., and Lovicario, M., 2019. BIM-based assessment metrics for the functional flexibility of building designs. Automation in Construction, 107 (November 2018).

Cicmil, S. and Marshall, D., 2005. Insights into collaboration at the project level: complexity, social interaction and procurement mechanisms. Building Research \& Information, 33 (6), 523-535. 
Cuneco, 2014. CCS Informationsniveauer. Center for productivitet i byggeriet, (december), 15.

Demaid, A. and Quintas, P., 2006. Knowledge across cultures in the construction industry: sustainability, innovation and design. Technovation, 26 (5-6), 603-610.

Dimyadi, J. and Amor, R., 2013. Automated Building Code Compliance Checking - Where is it at ? Proceedings of CIB WBC 2013, 172-185.

Dynamo BIM [online], 2018. Available from: http://dynamobim.org/ [Accessed 17 Mar 2016].

Eastman, C., Lee, J., Jeong, Y., and Lee, J., 2009. Automatic rule-based checking of building designs. Automation in Construction, 18 (8), 1011-1033.

Fan, S.-L., Chi, H.-L., and Pan, P.-Q., 2019. Rule checking Interface development between building information model and end user. Automation in Construction, 105 (October 2018), 102842.

Fish, A.N., 2012. Knowledge Automation.

Ghannad, P., Lee, Y.C., Dimyadi, J., and Solihin, W., 2019. Automated BIM data validation integrating openstandard schema with visual programming language. Advanced Engineering Informatics, 40 (September 2018), 14-28.

Green Building Council Denmark, 2014. DGNB System Denmark.

Green, T.R.G. and Petre, M., 1996. Usability Analysis of Visual Programming Environments: a 'cognitive dimensions' framework. Journal of Visual Languages and Computing.

Häußler, M., Esser, S., and Borrmann, A., 2021. Code compliance checking of railway designs by integrating BIM, BPMN and DMN. Automation in Construction, 121 (May 2020).

Hevner, A.R., March, S.T., Park, J., and Ram, S., 2004. Design Science in Information Systems Research. MIS Quarterly, 28 (1), 75-105.

Hjelseth, E., 2015. Foundations for BIM-based model checking systems. Norwegian University of Life Sciences.

Hjelseth, E., 2016. Classification of BIM-based Model checking concepts. Journal of Information Technology in Construction, 21 (July), 354-370.

Hoffman, R.R., Johnson, M., Bradshaw, J.M., and Underbrink, A., 2013. Trust in Automation. IEEE Intelligent Systems, (aprIL), 61-67.

Inagaki, T., Moray, N., and Itoh, M., 1998. Trust, Self-Confidence and Authority in Human-Machine Systems. IFAC Proceedings Volumes, 31 (26), 431-436.

Jewitt, C., 2012. An introduction to using video for research. National Centre for Research Methods Working Paper, 1-22.

Jick, T.D., 1979. Mixing Qualitative and Quantitative Methods: Triangulation in Action. Administrative Science Quarterly, 24 (4), 602.

Kaplan, B., Duchon, D., and Study, A.C., 1988. Combining Qualitative and Quantitative Information Systems, 12 (4), 571-586.

Kazi, A.S. and Koivuniemi, A., 2006. Sharing through social interaction: The case study of YIT Construction Ltd. In: A.S. Kazi and P. Wolf, eds. Real-Life Knowledge Management, Lessons from the field. KnowledgeBoard in collaboration with VTT - Technical Research Centre of Finland, 65-78.

Kim, H., Lee, J.-K., Shin, J., and Choi, J., 2018. Visual Language Approach to Representing KBimCode-based Korea Building Code Sentences for Automated Rule Checking. Journal of Computational Design and Engineering.

Koch, C., Paavola, S., and Buhl, H., 2019. Social science and construction - an uneasy and underused relation. Construction Management and Economics, 37 (6), 309-316.

Koncevics, R., Penicina, L., Gaidukovs, A., Dargis, M., Burbo, R., and Auzins, A., 2017. Comparative Analysis of Business Process Modelling Tools for Automated Compliance Management. Applied Computer Systems, 21 (May), 22-27.

Mclean, W., 2003. Inspector Discretion and Industrycompliance in streel level implementation of building codes. University of New Orleans Theses and Dissertations. University of new Orleans.

Miettinen, R. and Paavola, S., 2014. Beyond the BIM utopia: Approaches to the development and implementation of building information modeling. Automation in Construction, 43, 84-91.

Moray, N., Inagaki, T., and Itoh, M., 2000. Adaptive automation, trust, and self-confidence in fault management 
of time-critical tasks. Journal of experimental psychology Applied, 6 (1), 44-58.

Murphie, A. and Potts, J., 2017. Culture and technology. Macmillan International Higher Education.

Nawari, N.O., 2019. A Generalized Adaptive Framework (GAF) for automating code compliance checking. Buildings, 9 (4).

Nielsen, J., 2000. Why You Only Need to Test with 5 Users [online]. Nielsen Norman Group. Available from: https://www.nngroup.com/articles/why-you-only-need-to-test-with-5-users/ [Accessed 10 Oct 2017].

Nielsen, J. and Kaufmann, M., 1993. Usability Engineering.

Orlikowski, W.J., 1992. The Duality of Technology: Rethinking the Concept of Technology in Organizations. Organization Science, 3 (3), 398-427.

Parasuraman, R., Sheridan, T.B., and Wickens, C.D., 2000. A model for types and levels of human interaction with automation. IEEE transactions on systems, man, and cybernetics. Part A, Systems and humans : a publication of the IEEE Systems, Man, and Cybernetics Society, 30 (3), 286-297.

Preidel, C. and Borrmann, A., 2015a. Integrating Relational Algebra into a Visual Code Checking Language for Information Retrieval from Building Information Models. Icccbe, (June).

Preidel, C. and Borrmann, A., 2015b. Automated Code Compliance Checking Based on a Visual Language and Building Information Modeling. Proceedings of the International Symposium of Automation and Robotics in Construction, (JUNE).

Preidel, C. and Borrmann, A., 2016. TOWARDS CODE COMPLIANCE CHECKING ON THE BASIS OF A VISUAL PROGRAMMING LANGUAGE, 21 (July), 402-421.

Preidel, C. and Borrmann, A., 2017. Refinement of the visual code checking language for an automated checking of building information models regarding applicable regulations. In: Congress on Computing in Civil Engineering, Proceedings.

Preidel, C., Daum, S., and Borrmann, A., 2017. Data retrieval from building information models based on visual programming. Visualization in Engineering, 5 (1), 18.

Refvik, R., Skallerud, M., Slette, P.A., and Bjaaland, A., 2014. ByggNett - Status survey of solutions and issues relevant to the development of ByggNett.

Reichert, M. and Weber, B., 2012. Enabling Flexibility in Process-aware Information Systems Challenges, Paradigms, Technologies. 1st ed. Berlin Heidelberg: Springer.

Reinhardt, J. and Matthews, M., 2017. The Automation of BIM for Compliance Checking : a Visual Programming Approach. In: CITA BIM Gathering. Dublin 3: Dublin School of Architecture.

Sacks, R., Ma, L., Yosef, R., Borrmann, A., Daum, S., and Kattel, U., 2017. Semantic Enrichment for Building Information Modeling: Procedure for Compiling Inference Rules and Operators for Complex Geometry. Journal of Computing in Civil Engineering, 31 (6), 04017062.

Schimanski, C.P., Monizza, G.P., Marcher, C., and Matt, D.T., 2019. Pushing digital automation of configure-toorder services in small and medium enterprises of the construction equipment industry: A design science research approach. Applied Sciences (Switzerland), 9 (18), 1-22.

Tableau, 2018. Business Intelligence and Analytics Software [online]. Available from: https://www.tableau.com/ [Accessed 31 Oct 2018].

Whitley, K.N., 1996. Visual Programming Languages and the Empirical Evidence For and Against, (October).

Wood, H., Piroozfar, A., and Farr, E.R.P., 2013. Understanding complexity in the AEC industry. In: Proceedings of the 29th Annual Conference of the Association of Researchers in Construction Management (ARCOM). Reading, UK., 859-869. 